\title{
The long noncoding RNA LINC00312 induces lung adenocarcinoma migration and vasculogenic mimicry through directly binding YBX1
}

Zhenzi Peng ${ }^{1}$, Jun Wang ${ }^{1}$, Bin Shan ${ }^{2}$, Bin Li $i^{1}$, Wei Peng ${ }^{1}$, Yeping Dong ${ }^{1}$, Wenwen Shi', Wenyuan Zhao ${ }^{1}$, Dan He ${ }^{4}$, Minghao Duan', Yuanda Cheng ${ }^{3}$, Chunfang Zhang ${ }^{3}$ and Chaojun Duan ${ }^{1 *}$

\begin{abstract}
Vasculogenic mimicry (VM) gives rise to tumor neovascularization that is critical for tumor growth and metastasis. Long non-coding RNAs (IncRNAs) have been implicated in diverse and fundamental biological processes. LINC00312 is associated with lung adenocarcinoma. In this study, we found that LINC00312 induced migration, invasion and VM of lung cancer cells by direct binding to the transcription factor Y-Box Binding Protein 1 (YBX1). Moreover, we demonstrated that YBX1 is associated with different fragments within 0-2410 nt 5'region of LINC00312. In addition, LINC00312 is associated with VM in 124 lung adenocarcinoma clinical specimens. The results suggest that LINC00312 is a promising therapeutic and diagnostic target for lung adenocarcinoma.
\end{abstract}

Keywords: LINC00312, YBX1, Metastasis, Vasculogenic mimicry, Lung adenocarcinoma

\section{Main text}

Lung cancer is the most common cause of cancer related death in men and women. One of the most common types of lung cancer is NSCLC. Tumor neovascularization provides adequate blood and nutrition supply for tumor progression. Vasculogenic mimicry (VM) is one of the important sources of angiogenesis and nutritional supply in cancer [1]. The lncRNA metastasis-associated lung adenocarcinoma transcript 1 (MALAT-1) promotes the formation of VM in vitro, which suggests a role of lncRNA in angiogenesis [2]. We profiled lncRNA expression in lung adenocarcinoma (ADC) and identified LINC00312 as a differentially expressed lncRNA in our previous work [3, 4]. However, whether LINC00312 associated with VM in $\mathrm{ADC}$ remains unclear.

\footnotetext{
*Correspondence: duancjxy@126.com

${ }^{1}$ Institute of Medical Sciences, Key Laboratory of Cancer Proteomics of Chinese Ministry of Health, Xiangya Hospital, Central South University, Xiangya Road 87th, Changsha 410008, Hunan, People's Republic of China Full list of author information is available at the end of the article
}

Higher expression of LINC00312 was associated with metastasis of ADC patients

In this study, we measured the expression level of LINC00312 in 124 paired ADC tumor tissues and its adjacent non-tumor lung tissues using qRT-PCR. LINC00312 expression in patients with $\mathrm{ADC}$ metastasis was higher than patients without metastasis $(p<0.0001)$ (Fig. 1a). The clinicopathological analysis revealed that LINC00312 high expression was associated with lymph node metastasis $(p<0.001)$, distant metastasis $(p<0.001)$, tumor node metastasis (TNM) stage $(p=0.042)$ (Additional file 1: Table S1).

\section{Ectopic expression of LINC00312 promoted metastasis} and VM of ADC cells

To further characterize the role of LINC00312, we measured metastasis of ADC cells in vivo using bioluminescence imaging. The mice inoculated with the pIRES2-LINC00312 PC-9 overexpression cells exhibited more lung metastatic tumor nodules than the mice inoculated with the control PC-9 cells (Fig. 1b). Increased metastasis to the lung was confirmed by histological analysis. As expected, compared with the control group, pIRES2-LINC00312 PC-9 overexpression group showed 


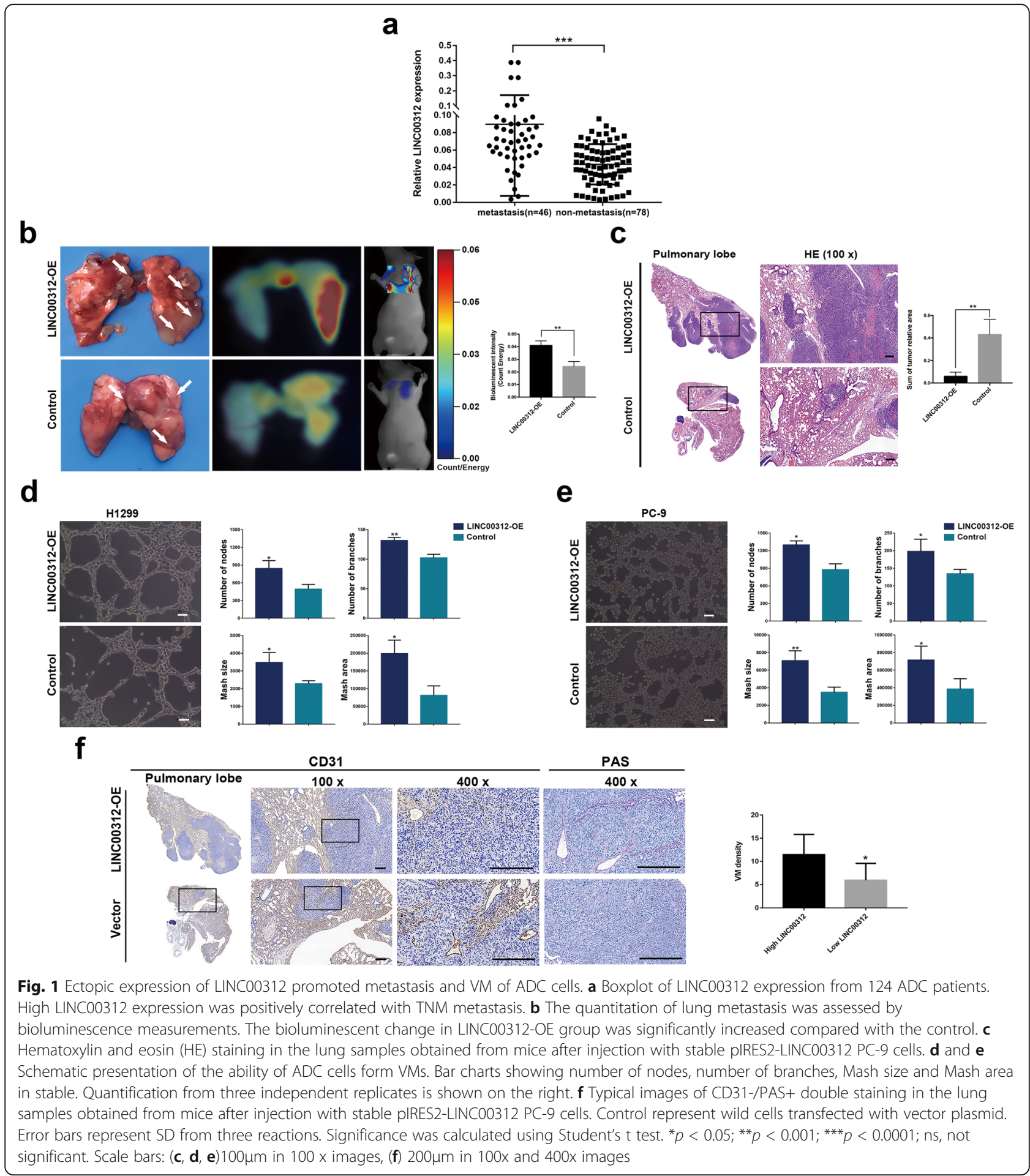

more lung metastatic nodules. (Fig. 1c). These results indicate that LINC00312 promotes metastasis in vivo. We further analyzed the effects of ectopic LINC00312 expression on tube formation of ADC cells (H1299 and PC-9) to gain insight into the potential role of LINC00312 in VM formation (Fig. 1 d, e). Our data revealed that the average VM density in tumor tissues formed in LINC00312 overexpression cells was significantly higher than that in control cells (Fig. 1f).

\section{Prognostic significance of LINC00312 expression in combination with VM density}

Next, we performed CD31 and PAS double staining to explore correlations between LINC00312 and VM in 124 


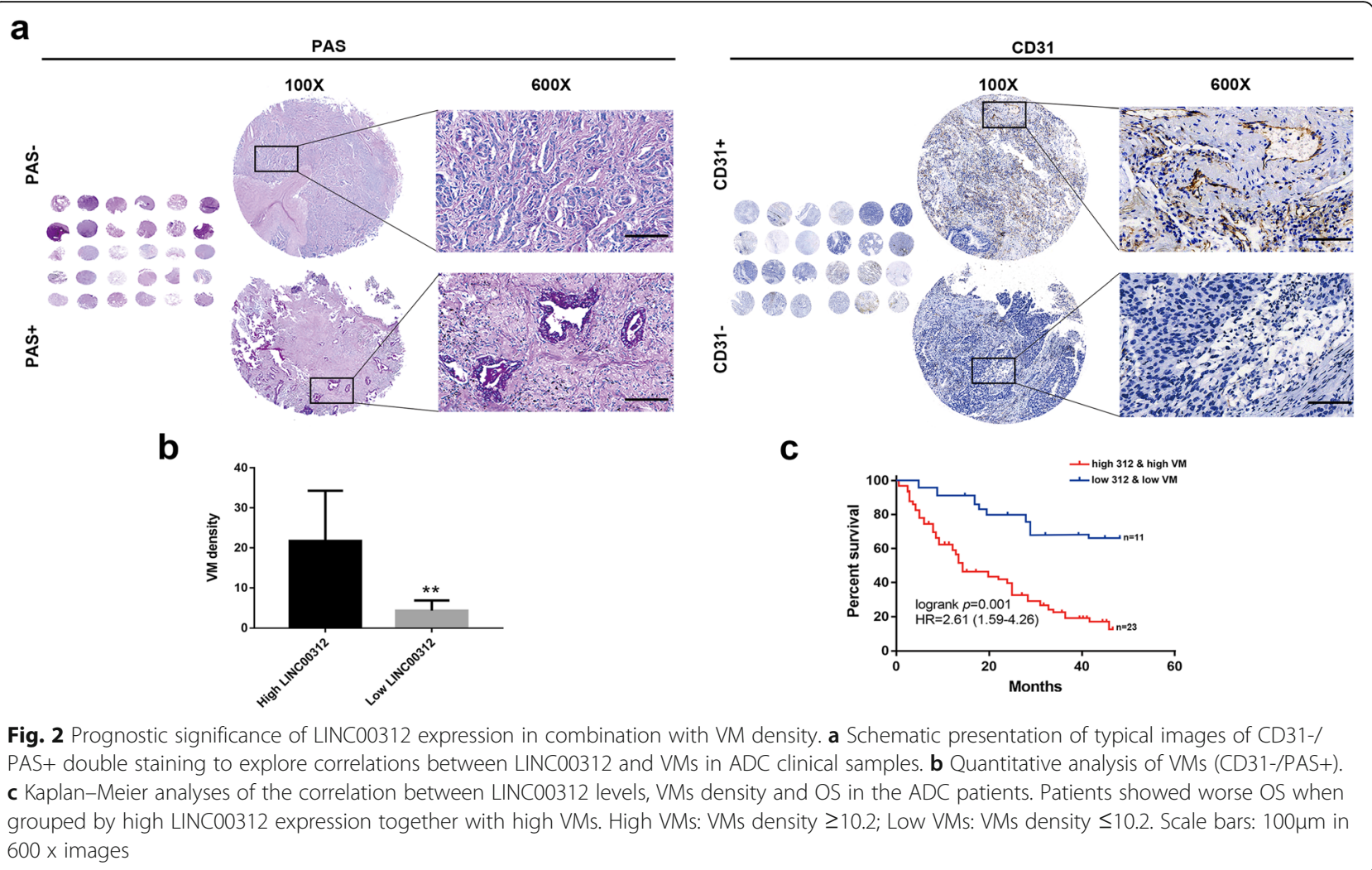

ADC clinical samples. The LINC00312 expression levels and clinicopathological analyses are summarized in Additional file 1: Table S1. We used CD31-/PAS+ as a criterium for VM [5]. Angiogenesis in malignant tumor tissues is associated with a poor clinical outcome in ADC patients, and the patients with VM exhibited greater tumor metastasis and a lower survival rate. Representative images of VM were shown in Fig. 2 a, b and quantified in Additional file 1: Table S1. Patients with simultaneous high levels of LINC00312 expression and high density of VM exhibited a relatively poor prognosis (Fig. 2c).

LINC00312 promotes migration and invasion of ADC cells $\mathrm{VM}$ is mediated by increased migratory and invasive abilities of cancer cells [6]. The positive correlation between LINC00312 levels and VM prompted us to explore the effects of this lncRNA on ADC cell migration and invasion. We examined the expression of LINC00312 in 6 ADC cell lines. LINC00312 expression was high in $\mathrm{H} 1703$ and H2009, low in PC-9, and intermediate in H1299, A549, and H1975 (Additional file 2: Figure S1A). We achieved significant overexpression and knockdown of LINC00312 PC-9 and H1299 cells as validated by RT-PCR, respectively. The PC-9 and H1299 variants were used to explore the functions of LINC00312 in lung cancer cells. To elucidate the mechanisms of LINC00312-mediated VM and metastasis of $\mathrm{ADC}$, we performed wound healing (Additional file 2:
Figure $\mathrm{S1B}$ ), transwell migration, and transwell invasion (Additional file 2: Figure S1C) assays in $\mathrm{H} 1299$ and PC-9 with LINC00312 overexpression or knockdown, respectively. The results demonstrated significantly increased migration and invasion as a result of LINC00312 overexpression. In accordance, knockdown of LINC00312 reduced migration and invasion of $\mathrm{ADC}$ cell lines.

\section{LINC00312 directly binds to YBX1}

A large number of lncRNAs function through binding to a protein partner. Fluorescent in situ hybridization were performed to examine subcellular localization of LINC00312. The results indicated that LINC00312 was present in the nucleus and cytoplasm (Fig. 3a). To identify the LINC00312 interacting proteins we performed RNA pull-down assay with biotinylated LINC00312, followed by mass spectrometry (MS). Our result indicated that two protein bands were specifically precipitated by LINC00312 in PC-9 in RNA pull-down assay (Fig. 3b). Western blotting was carried out on the protein samples precipitated by the LINC00312 RNA pulldown assays using the antibodies specific for the above candidates. Our results indicated that only YBX1 was precipitated by LINC00312 (Fig. 3c; Additional file 3: Table S2). To further confirm this interaction, we carried out RIP assay using a YBX1-specific antibody, followed by qRT-PCR using the primers specific for LINC00312. As expected, LINC00312 


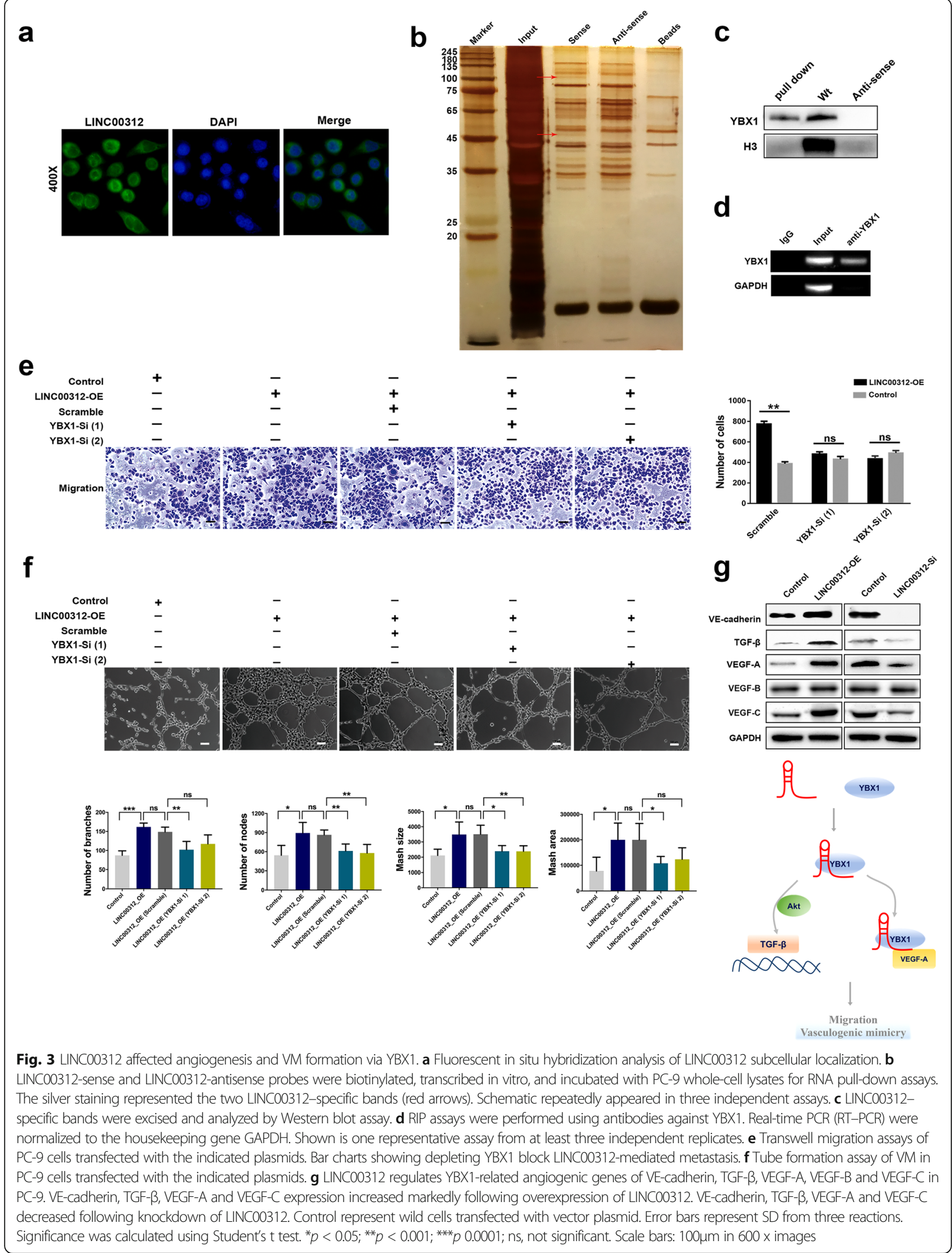


was enriched in the anti-YBX1 group, compared to control IgG group (Fig. 3d). These results indicated that YBX1, a transcription factor that regulates a range of gene expression, directly interacts with LINC00312.

To define the region of LINC00312 that was bound by YBX1, we generated three fragments (LINC00312 ${ }^{\mathrm{I}}$, LINC00312 ${ }^{\mathrm{II}}$, LINC00312 $2^{\mathrm{III}}$ ) according to its secondary structure (Additional file 4: Figure S2A). Pull-down assays demonstrated that YBX1 was enriched by both LINC00312 ${ }^{\mathrm{I}}$ and $\mathrm{LINC} 00312^{\mathrm{II}}$ (Additional file 4: Figure S2B). Western blotting on the proteins isolated from the RNA pulldown assays was used to determine whether YBX1 interacts with LINC00312 $^{\mathrm{I}}$ and LINC00312 ${ }^{\mathrm{II}}$ (Additional file 4: Figure S2C). Western blotting further confirmed the results of pulldown assay. Taken together, these data confirmed that YBX1 binds to LINC00312 ${ }^{\mathrm{I}}$ and LINC00312 ${ }^{\mathrm{II}}$ of LINC00312.

LINC00312 affected migration and VM formation via YBX1 A prooncogenic role for YBX-1 is suggested by its ability to promote migration and invasion of tumor cells [6]. Importantly, YBX1 up-regulates pro-angiogenic genes expression and plays a critical role in angiogenic switch $[7,8]$. Therefore, we hypothesized that LINC00312 induces migration of ADC cell lines through YBX1. To test this hypothesis, we analyzed migration and invasion when YBX1 was knockdown in the LINC00312 overexpression of PC-9. As expected, YBX1 knockdown partially blocked LINC00312-mediated increase in migration and invasion (Fig. 3e).

Moreover, VM is regarded as an important source for blood perfusion that supplies nutrients and oxygen to tumor growth and promotes cancer metastasis and progression. We postulate that LINC00312 induces ADC cell's VM via its binding to YBX1. Thus, we examined LINC00312-mediated VM when YBX1 was knockdown by RNAi. As expected, YBX1 knockdown blocked the LINC00312mediated VM (Fig. 3f). Quantification from three independent replicates is shown in number of branches, nodes, average mesh size area. These data demonstrate that LINC0 0312 promotes ADC cell's VM formation via YBX1.

LNC00312 affected many biological processes in ADC cells according to our RNA-seq results. The top GO terms and enriched KEGG pathways are related to YBX1-regulated angiogenic gene expression (Additional file 5: Figure S3).

It has been reported that YBX1 promotes TGF- $\beta$-induced migration via Akt activation. Elevated YBX1 expression increases cytosolic localization of VE-cadherin [9]. Moreover, YBX1 affects VEGF-A expression by binding to its promoter [10]. Hence, we further investigated whether LINC00312 affected their expression. As expected, western blot assay demonstrated that LINC00312 significantly increased VE-cadherin, TGF- $\beta$, VEGF-A and VEGF-C levels (Fig. 3g).

\section{Conclusion}

We demonstrated that LINC00312 induces lung adenocarcinoma metastasis and VM through direct binding to YBX1. Our study provides a significant advance in the current understanding of the dual roles for LINC00312 in lung cancer.

\section{Additional files}

Additional file 1: Table S1. Correlation between LINC00312 expression, VM density and clinicopathological parameters. (XLSX $11 \mathrm{~kb}$ )

Additional file 2: Figure S1. LINC00312 promotes migration and invasion of ADC cells. (TIF $9436 \mathrm{~kb}$ )

Additional file 3: Table S2. Proteins associated with biotinylated LINC00312 in RNA pull-down assay. (XLS 425 kb)

Additional file 4: Figure S2. A. Secondary structure of LINC00312. B. PC-9 whole-cell lysates pulled down with truncated LINC00312 or antisense probe. C. LINC00312 fragment-specific bands were excised and analyzed by Western blot assay. (TIF 1462 kb)

Additional file 5: Figure S3. Functional annotations of differentially expressed genes between LINC00312 stably overexpressed pIRES2LINC00312 and the vector control PC-9 cells. (A) Schematic presentation of top terms in the $\mathrm{GO}$ terms. (B) Schematic presentation of top terms in the KEGG terms. (TIF $4495 \mathrm{~kb}$ )

\section{Abbreviations}

IncRNAs: Long non-coding RNAs; MALAT-1: Metastasis-associated lung adenocarcinoma transcript 1; MS: Mass spectrometry; NSCLC: Non-small-cell lung cancer; TNM: Tumor node metastasis; VM: Vascular mimicry; YBX1: Y-Box Binding Protein 1

\section{Funding}

National Natural Science Foundation of China (81572281, 81702278, 81372515, 81402249)

\section{Availability of data and materials}

All data generated or analyzed in this study are included in this article [and its Additional files].

\section{Authors' contributions}

CJD procided direction and guidance throughout this manuscript. ZZP performed laboratory experiments and was a major contributor to the writing and editing of the manuscript. JW assisted in parts of experiments. $\mathrm{BS}$ reviewed and made significant revisions to the manuscript. All authors read and approved the final manuscript.

Ethics approval and consent to participate

Not applicable

Consent for publication

Not applicable

\section{Competing interests}

The authors declare that they have no competing interests.

\section{Publisher's note}

Springer Nature remains neutral with regard to jurisdictional claims in published maps and institutional affiliations.

\section{Author details}

${ }^{1}$ Institute of Medical Sciences, Key Laboratory of Cancer Proteomics of Chinese Ministry of Health, Xiangya Hospital, Central South University, Xiangya Road 87th, Changsha 410008, Hunan, People's Republic of China. 'Elison S Floyd College of Medicine, Washington State University, Spokane, WA 99201, USA. ${ }^{3}$ Department of Thoracic Surgery, Xiangya Hospital, Central South University, Changsha 410008, People's Republic of China. ${ }^{4}$ Hunan 
Cancer Hospital, The Affiliated Tumor Hospital of Xiangya Medical College, Central South University, Changsha 410008, People's Republic of China.

Received: 2 September 2018 Accepted: 19 November 2018

Published online: 23 November 2018

\section{References}

1. Qiao LQ, Liang NL, Zhang JD, Xie J, Liu FJ, Xu DG, Yu XS, Tian Y. Advanced research on vasculogenic mimicry in cancer. J Cell Mol Med. 2015;19:315-26.

2. Maria AS, Martin P. The role of long non-coding RNAs in osteosarcoma. Noncoding RNA. 2018:4:7.

3. Peng ZZ, Wang J, Shan B, Yuan FL, Li B, Dong YP, Peng W, Shi WW, Cheng YD, Gao Y, et al. Genome-wide analyses of long noncoding RNA expression profiles in lung adenocarcinoma. Sci Rep. 2017;7:15331.

4. Zhang W, Huang C, Gong Z, Zhao Y, Tang K, Li X, Fan S, Shi L, Li X, Zhang P, et al. Expression of LINC00312, a long intergenic non-coding RNA, is negatively correlated with tumor size but positively correlated with lymph node metastasis in nasopharyngeal carcinoma. J Mol Histol. 2013;44:545-54.

5. Junying S, Baocun S, Ran S, Dongwang Z, Zhao X, Zhang Y, Dong X, Na C, Li J, Fang L. HMGA2 promotes vasculogenic mimicry and tumor aggressiveness by upregulating Twist1 in gastric carcinoma. Sci Rep. 2017;7:2229-52.

6. Masaki S, Akira Y, Momoe I, Takeshi U, Yasuhiro TD, YooHyun S, Eiji K, Daisuke M, Seiji N. Twist1 and Y-box-binding protein-1 promote malignant potential in bladder cancer cells. BJU Int. 2011;108:E142-9.

7. Lasham A, Samuel W, Cao H, Patel R, Mehta R, Stern JL, Reid G, Woolley AG, Miller LD, Black MA, et al. YB-1, the E2F pathway, and regulation of tumor cell growth. J Natl Cancer Inst. 2012;104:133-46.

8. Annette L, Cristin GP, Adele GW, Sandra D, Antomy WB. YB-1: oncoprotein prognostic marker and therapeutic target? Biochem J. 2013;449:11-23.

9. Bin H, Eun BL, Jun C, Yosup K, Ho HJ. YB-1 overexpression promotes a TGF-beta1induced epithelial-mesenchymal transition via Akt activation. Biochem Biophys Res Commun. 2015;458:347-51.

10. Leeanne SC, Lidia L, Julie B, Julie H, Peter D, Andrew GB, Mathew AV, Gregory JG. Phosphorylation of cold shock domain/Y-box proteins by ERK2 and GSK3beta and repression of the human VEGF promoter. FEBS Lett. 2005;579:5372-8

Ready to submit your research? Choose BMC and benefit from:

- fast, convenient online submission

- thorough peer review by experienced researchers in your field

- rapid publication on acceptance

- support for research data, including large and complex data types

- gold Open Access which fosters wider collaboration and increased citations

- maximum visibility for your research: over $100 \mathrm{M}$ website views per year

At $\mathrm{BMC}$, research is always in progress.

Learn more biomedcentral.com/submissions 